\title{
AcrA dependency of the AcrD efflux pump in Salmonella enterica serovar Typhimurium
}

\begin{abstract}
Seiji Yamasaki ${ }^{1,2,3}$, Saya Nagasawa ${ }^{1,2,4}$, Mitsuko Hayashi-Nishino ${ }^{1}$, Akihito Yamaguchi ${ }^{2,3,4}$ and Kunihiko Nishino ${ }^{1,5}$
Multidrug efflux pumps belonging to the resistance-nodulation cell division (RND) family have major roles in the intrinsic and elevated resistance of Gram-negative bacteria to a wide range of compounds. RND efflux pumps require two other proteins to function: a membrane fusion protein (MFP) and an outer membrane protein. A recent study demonstrated that Salmonella enterica serovar Typhimurium has five RND efflux systems: AcrAB, AcrD, AcrEF, MdtABC and MdsABC. Most RND efflux system genes also code for an MFP in the same operon; however, an MFP gene is not located near acrD, and the MFP, with which AcrD functions, remains to be studied in detail. The aim of this study was to investigate the requirement of an MFP for the AcrD efflux system in this organism. When overproduced, AcrD significantly increased the resistance of the acrB mutant to oxacillin, cloxacillin, nafcillin, carbenicillin, sulbenicillin, aztreonam, sodium dodecyl sulfate and novobiocin. The increase in drug resistance caused by AcrD overproduction was completely suppressed by deleting the MFP gene, acrA, or the multifunctional outer membrane channel gene, tolC. Although the overexpression of acrD did not confer drug resistance to the $\triangle a c r A B$ strain, co-overexpression of $a c r D$ with $a c r A$ increased the multidrug resistance of this mutant. Our results indicate that the AcrA MFP and TolC outer membrane protein, in addition to their roles in the AcrB efflux system, are required for the function of the AcrD efflux pump in S. enterica serovar Typhimurium.
\end{abstract}

The Journal of Antibiotics (2011) 64, 433-437; doi:10.1038/ja.2011.28; published online 20 April 2011

Keywords: AcrA; AcrD; drug efflux pump; multidrug resistance; Salmonella enterica

\section{INTRODUCTION}

Multidrug efflux pumps cause serious problems in cancer chemotherapy and bacterial infection treatments. In bacteria, drug resistance is often associated with multidrug efflux pumps that decrease intracellular drug accumulation. ${ }^{1,2}$ These efflux pumps are classified into following five families on the basis of sequence similarity: (1) major facilitator; (2) resistance-nodulation cell division (RND); (3) small multidrug resistance; (4) multidrug and toxic compound extrusion; and (5) ATP-binding cassette. ${ }^{3}$ The pumps belonging to the RND family are particularly effective in producing resistance because they form a tripartite complex with the periplasmic proteins belonging to the membrane fusion protein (MFP) family and the outer membrane channels, facilitating direct efflux of drugs to the external medium. RND pumps often possess wide substrate specificity. ${ }^{1}$

Salmonella enterica is a pathogen that causes a variety of diseases in humans ranging from gastroenteritis to bacteremia and typhoid fever. ${ }^{4}$ In the 1990s, the prevalence of multidrug-resistant $S$. enterica increased dramatically in the UK, ${ }^{5,6} \mathrm{USA}^{7,8}$ and Canada. ${ }^{9}$ In addition, many countries have documented outbreaks associated with drugresistant Salmonella in poultry, beef and pork. ${ }^{10-14}$ Emerging resistance to antibiotics in Salmonella has been found in pathogens isolated from humans and animals and is thus a potentially serious public health problem. ${ }^{15,16}$

Determination of bacterial genome sequences enables us to trace putative drug resistance genes in bacteria. ${ }^{17,18}$ A recent study showed that $S$. enterica serovar Typhimurium has five putative RND transporter systems (see Figure 1): AcrAB, AcrD, AcrEF, MdtABC and MdsABC. ${ }^{19}$ In S. enterica, the function of all RND transporter systems requires TolC (outer membrane channel), except for MdsABC, which requires either $\mathrm{MdsC}$ or TolC for drug resistance. ${ }^{20}$ Four of these system genes, acrAB, acrEF, $m d t A B C$ and $m d s A B C$ also code for an MFP ( $a c r A, a c r E, m d t A$ and $m d s A$, respectively) in the same operon (Figure 1). However, an MFP gene is not located near $\operatorname{acrD}$, and the MFP, with which AcrD functions, remains to be studied in detail (Figure 1). Previously, we reported that the overproduction of AcrD conferred multidrug resistance to the $\triangle a c r B$ mutant against novobiocin, sodium dodecyl sulfate (SDS) and sodium deoxycholate; ${ }^{19}$ however, its role in resistance against $\beta$-lactam antibiotics and requirement of MFP have not been elucidated.

In this study, we describe that AcrD contributes to Salmonella resistance against $\beta$-lactam antibiotics including oxacillin, cloxacillin, nafcillin, carbenicillin, sulbenicillin and aztreonam. In addition to the

${ }^{1}$ Laboratory of Microbiology and Infectious Diseases, Institute of Scientific and Industrial Research, Osaka University, Ibaraki, Osaka, Japan; ${ }^{2}$ Department of Cell Membrane Biology, Institute of Scientific and Industrial Research, Osaka University, Ibaraki, Osaka, Japan; ${ }^{3}$ Graduate School of Pharmaceutical Sciences, Osaka University, Suita, Japan; ${ }^{4}$ School of Pharmaceutical Sciences, Osaka University, Suita, Japan and 5PRESTO, Japan Science and Technology Agency, Chiyodaku, Tokyo, Japan

Correspondence: Dr K Nishino, Laboratory of Microbiology and Infectious Diseases, Institute of Scientific and Industrial Research, Osaka University, 8-1 Mihogaoka, Ibaraki, Osaka 567-0047, Japan.

E-mail: nishino@sanken.osaka-u.ac.jp

Received 10 January 2011; revised 9 March 2011; accepted 11 March 2011; published online 20 April 2011 

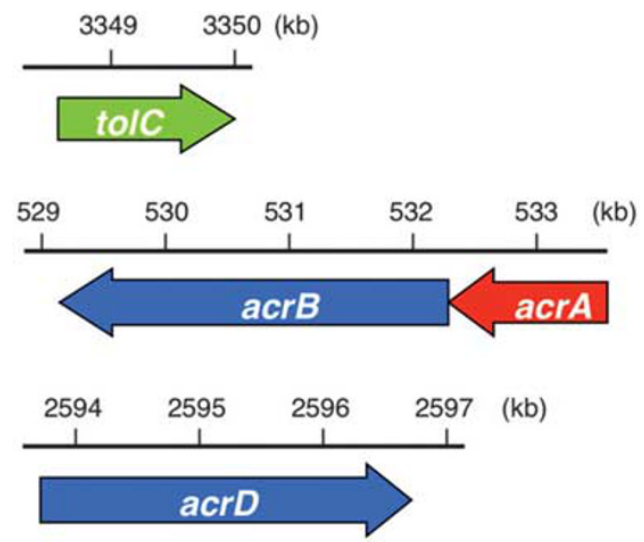

(kb)
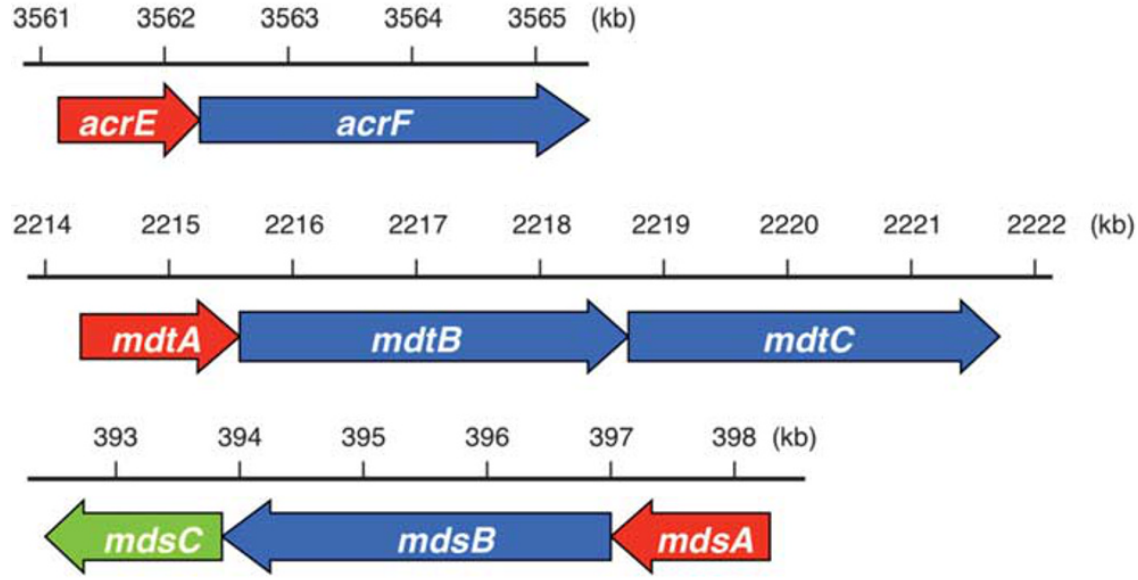

Figure 1 Resistance-nodulation cell division (RND) transporter system genes encoded in the $S$. enterica serovar Typhimurium genome. Chromosomal positions of genes encoding RND drug transporters, outer membrane proteins and membrane fusion proteins (MFPs) are indicated by 'kb' (kilobase pair) in the S. enterica serovar Typhimurium strain LT2 genome. ${ }^{29}$ Figure was modified from Nishino et al. (2006). ${ }^{19}$ Arrows correspond to the lengths and directions of the genes.

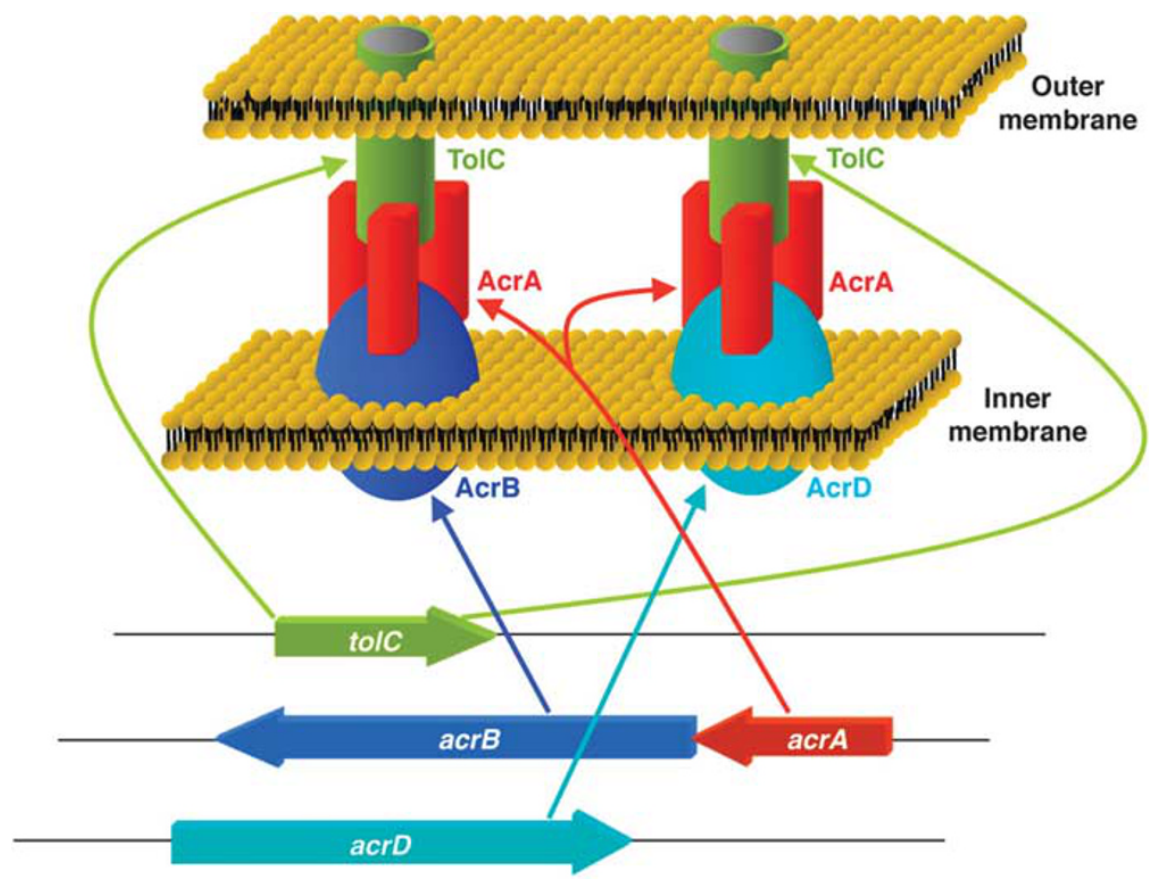

Figure 2 Model for AcrA usage as an membrane fusion protein (MFP) by the AcrAB-TolC and AcrD efflux systems in S. enterica. In S. enterica, AcrA functions with AcrB and AcrD efflux pumps, resulting in an increase in multidrug resistance. 
role of AcrA as MFP in the AcrAB multidrug efflux pump, we report that AcrA is required for the multidrug resistance conferred by the AcrD efflux pump (Figure 2). The results suggest multiple roles of AcrA as an MFP in S. enterica.

\section{MATERIALS AND METHODS}

Bacterial strains, plasmids and growth conditions

The bacterial strains and plasmids used in this study are listed in Table 1. The S. enterica serovar Typhimurium strains were derived from the wildtype strain ATCC 14028s (ref. 21). These strains were grown at $37^{\circ} \mathrm{C}$ in Luria-Bertani (LB) broth. ${ }^{22}$

\section{Construction of gene deletion mutants}

To construct the $\triangle a c r B$ mutant NKS1202, the kanamycin resistance marker of the $\triangle a c r B:: \mathrm{Km}^{\mathrm{R}}$ strain EG16565 (ref. 19) was eliminated using the pCP20 plasmid as described previously. ${ }^{23}$ To construct the $\triangle a c r A B \triangle a c r D$ mutant NKS1245, P22 phages isolated from the $\triangle a c r A B:: \mathrm{Km}^{\mathrm{R}}$ strain NKS147 and the $\triangle a c r D:: \mathrm{Cm}^{\mathrm{R}}$ strain EG16567 (ref. 19) were transduced to the wild-type strain ATCC 14028s, and kanamycin and chloramphenicol resistance markers were eliminated using the pCP20 plasmid. To construct the $\triangle a c r A B$ strain NKS234 or the $\Delta$ tolC strain NKS233, gene disruption was performed as described by

Table 1 S. enterica strains and plasmids used in this study

\begin{tabular}{|c|c|c|}
\hline $\begin{array}{l}\text { Strain or } \\
\text { plasmid }\end{array}$ & Characteristics & $\begin{array}{l}\text { Source or } \\
\text { reference }\end{array}$ \\
\hline \multicolumn{3}{|l|}{ Strains } \\
\hline ATCC $14028 \mathrm{~s}$ & S. enterica serovar Typhimurium wild type & 21 \\
\hline NKS1202 & $\Delta a c r B$ & This study \\
\hline NKS1254 & $\Delta a c r B / p A C Y C 177 \mathrm{Km}^{\mathrm{R}}$ & This study \\
\hline NKS1255 & $\Delta a c r B / p a c r A$ & This study \\
\hline NKS1235 & $\Delta$ acrB/pHSG398 & This study \\
\hline NKS1236 & $\Delta a c r B / p a c r D$ & This study \\
\hline NKS234 & $\triangle a c r A B$ & This study \\
\hline NKS1252 & $\triangle a c r A B / p A C Y C 177 \mathrm{Km}^{\mathrm{R}}$ & This study \\
\hline NKS1238 & $\Delta$ acrAB/pHSG398 & This study \\
\hline NKS1253 & $\Delta a c r A B / p A C Y C 177 \mathrm{Km}^{\mathrm{R}}+\mathrm{pHSG} 398$ & This study \\
\hline NKS1240 & $\Delta a c r A B / p a c r A$ & This study \\
\hline NKS1241 & $\Delta a c r A B / p a c r D$ & This study \\
\hline NKS1242 & $\Delta a c r A B / p a c r A+p a c r D$ & This study \\
\hline NKS1245 & $\Delta a c r A B \Delta a c r D$ & This study \\
\hline NKS1246 & $\triangle a c r A B \Delta a c r D / p A C Y C 177 \mathrm{Km}^{\mathrm{R}}$ & This study \\
\hline NKS 1247 & $\Delta a c r A B \Delta a c r D / p H S G 398$ & This study \\
\hline NKS1248 & $\Delta a c r A B \Delta a c r D / p A C Y C 177 \mathrm{Km}^{\mathrm{R}}+\mathrm{pHSG} 398$ & This study \\
\hline NKS1249 & $\Delta a c r A B \Delta a c r D / p a c r A$ & This study \\
\hline NKS1250 & $\Delta a c r A B \Delta a c r D / p a c r D$ & This study \\
\hline NKS1251 & $\Delta$ acrAB $\Delta a c r D / p a c r A+$ pacrD & This study \\
\hline NKS233 & $\Delta$ tolC & This study \\
\hline NKS1243 & $\Delta$ tolC/pHSG398 & This study \\
\hline NKS1244 & $\Delta$ tolC/pacrD & This study \\
\hline \multicolumn{3}{|l|}{ Plasmids } \\
\hline pKD46 & Red recombinase expression plasmid, $A p^{R}$ & 23 \\
\hline pCP20 & reppsc101 $^{\text {ts }} \mathrm{Ap}^{\mathrm{R}} \mathrm{Cm}^{\mathrm{R}}$ cl857ëP $\mathrm{P}$ flp & 23 \\
\hline pACYC177 & vector; $A p^{R}, \mathrm{Km}^{\mathrm{R}}$ & MBI Fermentas \\
\hline $\mathrm{pACYC} 177 \mathrm{Km}^{\mathrm{R}}$ & vector; $\mathrm{Km}^{\mathrm{R}}$ & This study \\
\hline pacrA & 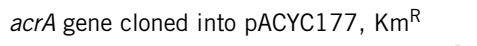 & This study \\
\hline pHSG398 & $\begin{array}{l}\text { vector; derivative of pUC18 containing } \mathrm{Cm}^{\mathrm{R}} \\
\text { in place of } A p^{R}\end{array}$ & Takara Bio Inc. \\
\hline pacrD & acrD gene cloned into pHSG398, $\mathrm{Cm}^{\mathrm{R}}$ & This study \\
\hline
\end{tabular}

Datsenko and Wanner. ${ }^{23}$ The following oligonucleotide primers were used for the construction of the mutants:

acrA-P2

(5'-ACCATTGACCAATTTGAAATCGGACACTCGAGGTTTACATCATATGA ATATCCTCCTTAG-3');

acrB-P1

(5'-AAAAAAGGCCGCTTGCGCGGCCTTATCAACAGTGAGCAAAGTGTA

GGCTGGAGCTGCTTC-3')

tolC-P2

(5'-TACAAATTGATCAGCGCTAAATACTGCTTCACAACAAGGACATATGA ATATCCTCCTTAG-3');

and $t o l C-\mathrm{P} 1$

(5'-GGGCACAGGTCTGATAAGCGCAGCGCCAGCGAATAACTTAGTGTAG GCTGGAGCTGCTTC-3').

The chloramphenicol resistance gene, cat, or the kanamycin resistance gene, $a p h$, flanked by Flp recognition sites, was amplified by PCR using the aforementioned primers. The resulting PCR products were used to transform the recipient ATCC 14028s strain harboring the pKD46 plasmid expressing Red recombinase. The chromosomal structure of the mutated loci was verified by PCR, as described previously. ${ }^{23}$ cat and aph were eliminated using the pCP20 plasmid, as described previously. ${ }^{23}$

\section{Plasmid construction}

acrA was amplified from ATCC 14028s genomic DNA using the primers 5'-CGCAGTACTATGTCGGTGAATTTACAGGCG-3' and 5'-CGCGGATCCGT CTTAACGGCTCCTGTTTAA-3', which introduced ScaI and BamHI sites (underlined in the primer sequences above), respectively. The PCR fragment contained a region from $141 \mathrm{bp}$ upstream to $22 \mathrm{bp}$ downstream of acrA. This fragment was digested with $\mathrm{ScaI}$ and $\mathrm{Bam} \mathrm{HI}$ and cloned into the corresponding sites of pACYC177, where an ampicillin resistance gene was located. The resulting plasmid, pacrA, carried acrA instead of the ampicillin marker (Table 1). acrD was amplified using the primers $5^{\prime}$-CGCGGATCCCATTA TCTCCTTTATTTCTCC- $3^{\prime}$ and $5^{\prime}$-CGCGCATGCTTATTTCGGGCGCGGCTT CAG-3', which introduced BamHI and SphI sites (underlined in the primer sequences above), respectively. The PCR fragment contained a region from $100 \mathrm{bp}$ upstream to the stop codon of $a c r D$. This fragment was digested with BamHI and SphI and cloned into the corresponding sites of pHSG398 to produce pacrD (Table 1). To obtain pACYC177 without the ampicillin marker, pACYC177 was digested at two sites with Psp1406 (AclI) and self-ligated. The resulting vector was named $\mathrm{pACYC} 177 \mathrm{Km}^{\mathrm{R}}$.

\section{Determination of MICs of toxic compounds}

The antibacterial activities of various agents were determined on LB agar ( $1 \%$ tryptone, $0.5 \%$ yeast extract, $0.5 \% \mathrm{NaCl}$ ) plates containing oxacillin, cloxacillin, nafcillin, carbenicillin, sulbenicillin, aztreonam, SDS, or novobiocin (Sigma, St Louis, MO, USA) at various concentrations. Agar plates were made by the twofold agar dilution technique, as described previously. ${ }^{24}$ To determine the MICs, bacteria were grown in LB broth at $37^{\circ} \mathrm{C}$ overnight, diluted into the same medium, and then tested at a final inoculum size of $10^{5} \mathrm{CFU}$ per $\mu$ by using a multipoint inoculator (Sakuma Seisakusyo, Tokyo, Japan) after incubation at $37^{\circ} \mathrm{C}$ for $20 \mathrm{~h}$. The MIC was the lowest concentration of a compound that inhibited cell growth.

\section{RESULTS}

Effect of $a c r D$ overexpression on the multidrug resistance of the $\Delta a c r B$ strain

To investigate the role of the AcrD drug efflux pump in multidrug resistance, acrD in S. enterica serovar Typhimurium ATCC14028s was cloned into the vector pHSG398, and the constructed plasmid was transformed into the $\triangle a c r B$ strain. Compared with the wild-type strain, the $\triangle a c r B$ strain showed increased susceptibility to all eight antimicrobial agents and chemical compounds tested (Table 2). The transformant $(\Delta a c r B / p a c r D)$ showed increased resistance to SDS and novobiocin as we previously reported. ${ }^{19,20}$ Because we had previously cloned $a c r D$ into the pUC118 vector, which had the $\beta$-lactamase gene 
Table 2 Susceptibility of $S$. enterica strains to toxic compounds

\begin{tabular}{|c|c|c|c|c|c|c|c|c|}
\hline \multirow[b]{2}{*}{ Strain } & \multicolumn{8}{|c|}{$M I C\left(\mu g m I^{-1}\right)$} \\
\hline & $O X A$ & $C L X$ & NAF & $C A R$ & $S B$ & ATM & $S D S$ & NOV \\
\hline$\Delta a c r B$ & 2 & 4 & 8 & 1 & 2 & 0.031 & 256 & 2 \\
\hline$\Delta a c r B / p A C Y C 177 K^{R}$ & 2 & 4 & 8 & 1 & 2 & 0.031 & 256 & 2 \\
\hline$\Delta$ acrB/pacrA & 2 & 4 & 8 & 1 & 2 & 0.031 & 256 & 2 \\
\hline$\triangle a c r A B$ & 1 & 2 & 4 & 0.5 & 1 & 0.031 & 256 & 2 \\
\hline$\triangle a c r A B / p A C Y C 177 K^{R}$ & 1 & 2 & 4 & 0.5 & 1 & 0.031 & 256 & 2 \\
\hline$\triangle a c r A B / p H S G 398$ & 1 & 2 & 4 & 0.5 & 1 & 0.031 & 256 & 2 \\
\hline$\triangle a c r A B / p A C Y C 177 K^{R}+$ pHSG398 & 1 & 2 & 4 & 0.5 & 1 & 0.031 & 256 & 2 \\
\hline$\triangle a c r A B / p a c r A$ & 2 & 4 & 8 & 1 & 2 & 0.031 & 256 & 2 \\
\hline$\triangle a c r A B / p a c r D$ & 1 & 2 & 4 & 0.5 & 1 & 0.031 & 256 & 2 \\
\hline$\Delta$ acrAB $\triangle a c r D / p A C Y C 177 K^{R}+p H S G 398$ & 1 & 2 & 4 & 0.5 & 1 & 0.031 & 256 & 2 \\
\hline$\Delta a c r A B \Delta a c r D / p a c r A$ & 1 & 2 & 4 & 0.5 & 1 & 0.031 & 256 & 2 \\
\hline$\Delta a c r A B \Delta a c r D / p a c r D$ & 1 & 2 & 4 & 0.5 & 1 & 0.031 & 256 & 2 \\
\hline$\triangle a c r A B \Delta a c r D / p a c r A+p a c r D$ & 16 & 32 & 128 & 8 & 64 & 0.25 & 65536 & 4 \\
\hline$\Delta$ tolc & 0.25 & 0.25 & 0.5 & 0.5 & 1 & 0.031 & 32 & 0.5 \\
\hline$\Delta$ tolC/pHSG398 & 0.25 & 0.25 & 0.5 & 0.5 & 1 & 0.031 & 32 & 0.5 \\
\hline$\Delta$ tolC/pacrD & 0.25 & 0.25 & 0.5 & 0.5 & 1 & 0.031 & 32 & 0.5 \\
\hline
\end{tabular}

Abbreviations: ATM, aztreonam; CLX, cloxacillin; CAR, carbenicillin; NAF, nafcillin; NOV, novobiocin; OXA, oxacillin; SB, sulbenicillin; SDS, sodium dodecyl sulfate. Values in bold are larger than those of the corresponding parental strains. MIC determinations were repeated at least three times.

as a marker, the effect of $a c r D$ overexpression on the $\beta$-lactam resistance of $S$. enterica was unknown. ${ }^{19,20}$ In this study, acrD was cloned into the pHSG398 vector that had a chloramphenicol resistance marker. In addition to SDS and novobiocin, the pacrD plasmid conferred resistance to $\beta$-lactam antibiotics such as oxacillin, cloxacillin, nafcillin, carbenicillin, sulbenicillin and aztreonam. These data indicate that the AcrD efflux system confers multidrug resistance to S. enterica against SDS, novobiocin and $\beta$-lactam antibiotics.

\section{Effect of $a c r D$ overexpression on the drug susceptibility of the $\triangle a c r A B$ or $\triangle$ tolC strains}

We previously reported that only the AcrAB-TolC efflux system was expressed under laboratory conditions, whereas other efflux systems required additional cues for detectable expression. ${ }^{19}$ Thus, to determine the components required for the function of the AcrD efflux system, we focused on the outer membrane channel, TolC and the MFP, AcrA. We constructed the $\triangle t o l C$ and $\triangle a c r A B$ mutants of $S$. enterica, and the plasmid carrying acrD was transformed into these mutants (Table 1). In contrast to the results of the $\Delta$ acrB mutant, overexpression of $a c r D$ did not confer multidrug resistance to $\triangle t o l C$ and $\triangle a c r A B$ mutants (Table 2). These data suggest that the multidrug resistance conferred by $A c r D$ overproduction is dependent on the presence of TolC and AcrA proteins.

\section{Effect of acrA overexpression on the drug susceptibility} of $S$. enterica

In the drug susceptibility tests, we observed that the $\triangle a c r A B$ strain was slightly (twofold change) susceptible to oxacillin, cloxacillin, nafcillin, carbenicillin and sulbenicillin compared with the $\triangle a c r B$ strain
(Table 2). This phenotype was complemented with the plasmid carrying acrA (Table 2). The $\triangle a c r A B$ strain harboring the pacrA plasmid behaved like the $\triangle a c r B$ strain. This result suggests the possibility that AcrA may be required for other drug resistance factors in addition to AcrB. The aforementioned results obtained using the plasmid carrying $a c r D$ and the $\triangle a c r A B$ strain suggests that AcrD may require AcrA for its function. To determine whether the function of chromosomally encoded AcrD causes the difference in MICs between the $\triangle a c r B$ and $\triangle a c r A B$ strains, the plasmid carrying acrA was transformed into the $\triangle a c r A B$ and $\triangle a c r A B \triangle a c r D$ strains (Table 1). The drug susceptibility of the $\triangle a c r A B \triangle a c r D$ strain was similar to that of the $\triangle a c r A B$ strain (Table 2). In contrast to the case of the $\triangle a c r A B$ mutant, the plasmid carrying acrA did not increase the drug resistance of the $\triangle a c r A B \triangle a c r D$ mutant. These data suggest that increased susceptibility of the $\triangle a c r A B$ strain compared with the $\triangle a c r B$ strain is due to loss of function of the chromosomally expressed AcrD efflux pump along with the deletion of $a c r A$.

\section{Effect of the co-expression of acrD and acrA on the drug susceptibility of $S$. enterica}

The aforementioned results suggest the possibility that the AcrD efflux pump requires AcrA for its function as an MFP. To verify whether AcrA functions as the MFP of the AcrD efflux system, pacrD and pacrA were co-transformed into the $\triangle a c r A B$ strain (Table 1). Interestingly, co-expression of $a c r A$ and $a c r D$ significantly conferred drug resistance to the $\triangle a c r A B$ strain against all eight compounds tested, whereas overexpression of $a c r D$ alone did not confer any resistance to this strain. In addition, co-expression of $\operatorname{acr} A$ and $\operatorname{acr} D$ conferred multidrug resistance to the $\triangle a c r A B \triangle a c r D$ strain. These 
results indicate that AcrA (an MFP) is required for the function of the AcrD efflux pump and contributes to AcrD-mediated multidrug resistance (Figure 2).

\section{DISCUSSION}

In this study, we identified that the AcrD efflux system confers resistance to various compounds including $\beta$-lactam antibiotics and requires AcrA as an MFP in S. enterica. In a previous study, we determined that the overexpression of $a c r D$ conferred resistance to the $\triangle a c r B$ strain of $S$. enterica against SDS and novobiocin. However, the effect of acrD on Salmonella susceptibility to $\beta$-lactam antibiotics had not been investigated because acrD was cloned into the pUC118 vector, which had the $\beta$-lactamase gene as a marker. ${ }^{19}$ Here, we demonstrated that the plasmid carrying acrD conferred resistance not only to SDS and novobiocin, but also to oxacillin, cloxacillin, nafcillin, carbenicillin, sulbenicillin and aztreonam in the $\Delta a c r B$ mutant. Although this plasmid did not increase the multidrug resistance of the $\triangle a c r A B$ mutant, the co-expression of $a c r D$ with $a c r A$ increased the multidrug resistance of this mutant. Furthermore, in contrast to the result that the $\triangle a c r A B$ mutant complemented with pacrA behaved like $\triangle a c r B$ mutant, the plasmid carrying $a c r A$ did not increase the drug resistance of the $\triangle a c r A B \triangle a c r D$ mutant. These data suggest that chromosomally expressed $\mathrm{AcrD}$, despite the low expression level, ${ }^{19}$ induces intrinsic multidrug resistance in $S$. enterica. In Escherichia coli, it was suggested that the function of the AcrD system depends on AcrA, although a co-expression study of acrD with acrA was not previously performed. ${ }^{25,26}$ In this study, on the basis of the results of co-expression of $a c r D$ with $a c r A$, we revealed that AcrD of S. enterica requires AcrA for its function. In addition to the role of AcrA in the AcrAB-TolC system, our study showed that it was required for the function of the AcrD efflux system in S. enterica (Figure 2).

One possibility for AcrD using AcrA as an MFP when it is coded in a different operon is that AcrD has a compensatory role when AcrB function is disrupted. In other words, AcrD may form a complex with AcrA and TolC when mutations occur in AcrB and compensate for the lost function of AcrAB-TolC multidrug efflux system. Another possibility is that AcrA contributes to different biological functions by forming complexes with two different RND pumps, AcrB and AcrD. As described previously, ${ }^{1}$ AcrB is an important factor for bacterial multidrug resistance. It was recently reported that $A c r D$ contributes to resistance against metals such as copper or zinc in addition to its role in multidrug resistance. ${ }^{27,28}$ Because AcrA is required for the functioning of AcrD, AcrA may be involved in AcrD-mediated metal resistance. Such a functional network of multidrug efflux pumps may contribute to bacterial adaptation to various environmental conditions.

Further investigation of the cooperation of multidrug efflux systems is required to elucidate their biological significance in natural environments. Such investigations may provide further insights into the role of these systems in cell physiology.

\section{ACKNOWLEDGEMENTS}

SY is supported by research fellowships from the Tosoh Scholarship Foundation and the Yoshida Scholarship Foundation. This research was supported by research aid from the Sumitomo Foundation (to MH-N), the Institute for Fermentation (to MH-N), and the Program for Promotion of Fundamental Studies in Health Sciences of the National Institute of Biomedical Innovation (to AY); a grant from the Ministry of Education, Culture, Sports, Science and Technology of Japan (to KN); Grants-in-Aid for Young Scientists (S) (to KN), Research Activity Start-up (to MH-N), and Scientific Research (S) (to AY) from the Japan Society for the Promotion of Science; the program HISHO the Top Thirty Young Researchers of Osaka University (to KN); PRESTO (to KN),
Japan Science and Technology Agency; Funding Program for Next Generation World-Leading Researchers (to KN), Government of Japan.

1 Nikaido, H. Multidrug efflux pumps of gram-negative bacteria. J. Bacteriol. 178, 5853-5859 (1996)

2 Zgurskaya, H. I. \& Nikaido, H. Multidrug resistance mechanisms: drug efflux across two membranes. Mol. Microbiol. 37, 219-225 (2000).

3 Nishino, K., Nikaido, E. \& Yamaguchi, A. Regulation and physiological function of multidrug efflux pumps in Escherichia coli and Salmonella. Biochim. Biophys. Acta. 1794, 834-843 (2009).

4 Scherer, C. A. \& Miller, S. I. Molecular pathogenesis of Salmonella. In Principles of Bacterial Pathogenesis (ed. Groisman, E.A.) 266-333 (Academic Press, New York, 2001).

5 Threlfall, E. J., Frost, J. A., Ward, L. R. \& Rowe, B. Increasing spectrum of resistance in multiresistant Salmonella typhimurium. Lancet 347, 1053-1054 (1996).

6 Threlfall, E. J., Ward, L. R., Skinner, J. A. \& Rowe, B. Increase in multiple antibiotic resistance in nontyphoidal salmonellas from humans in England and Wales: a comparison of data for 1994 and 1996. Microb. Drug Resist. 3, 263-266 (1997).

7 Hosek, G., Leschinsky, D. D., Irons, S. \& Safranek, T. J. Multidrug-resistant Salmonella serotype Typhimurium-United States, 1996. Morb. Mortal. Wkly. Rep. 46, 308-310 (1997).

8 Glynn, M. K. et al. Emergence of multidrug-resistant Salmonella enterica serotype typhimurium DT104 infections in the United States. N. Engl. J. Med. 338, 1333-1338 (1998).

9 Ng, L. K., Mulvey, M. R., Martin, I., Peters, G. A. \& Johnson, W. Genetic characterization of antimicrobial resistance in Canadian isolates of Salmonella serovar Typhimurium DT104. Antimicrob. Agents Chemother. 43, 3018-3021 (1999).

10 Davies, A. O'Neill, P., Towers, L. \& Cooke, M. An outbreak of Salmonella typhimurium DT104 food poisoning associated with eating beef. Commun. Dis. Rep. CDR Rev. 6, R159-R162 (1996).

11 Cody, S. H. et al. Two outbreaks of multidrug-resistant Salmonella serotype Typhimurium DT104 infections linked to raw-milk cheese in Northern California. JAMA 281, 1805-1810 (1999).

12 Grein, T., O'Flanagan, D., McCarthy, T. \& Bauer, D. An outbreak of multidrug-resistant Salmonella typhimurium food poisoning at a wedding reception. Ir. Med. J. 92, 238-241 (1999).

13 Molbak, K. et al. An outbreak of multidrug-resistant, quinolone-resistant Salmonella enterica serotype typhimurium DT104. N. Engl. J. Med. 341, 1420-1425 (1999).

14 Villar, R. G. et al. Investigation of multidrug-resistant Salmonella serotype typhimurium DT104 infections linked to raw-milk cheese in Washington State. JAMA 281, 1811-1816 (1999).

15 Cloeckaert, A. \& Chaslus-Dancla, E. Mechanisms of quinolone resistance in Salmonella. Vet. Res. 32, 291-300 (2001).

16 Piddock, L. J. Fluoroquinolone resistance in Salmonella serovars isolated from humans and food animals. FEMS Microbiol. Rev. 26, 3-16 (2002).

17 Paulsen, I. T., Sliwinski, M. K. \& Saier, M. H. Jr Microbial genome analyses: global comparisons of transport capabilities based on phylogenies, bioenergetics and substrate specificities. J. Mol. Biol. 277, 573-592 (1998).

18 Paulsen, I. T., Nguyen, L., Sliwinski, M. K., Rabus, R. \& Saier, M. H. Jr Microbial genome analyses: comparative transport capabilities in eighteen prokaryotes. J. Mol. Biol. 301, 75-100 (2000).

19 Nishino, K., Latifi, T. \& Groisman, E. A. Virulence and drug resistance roles of multidrug efflux systems of Salmonella enterica serovar Typhimurium. Mol. Microbiol. 59, 126-141 (2006).

20 Horiyama, T., Yamaguchi, A. \& Nishino, K. TolC dependency of multidrug efflux systems in Salmonella enterica serovar Typhimurium. J. Antimicrob. Chemother. 65, 1372-1376 (2010).

21 Fields, P. I., Swanson, R. V., Haidaris, C. G. \& Heffron, F. Mutants of Salmonella typhimurium that cannot survive within the macrophage are avirulent. Proc. Natl Acad. Sci. USA 83, 5189-5193 (1986).

22 Sambrook, J., Fritsch, E. F. \& Maniatis, T. Molecular Cloning: A Laboratory Manual. 2nd edn. (Cold Spring Harbor Laboratory, Cold Spring Harbor, NY, 1989).

23 Datsenko, K. A. \& Wanner, B. L. One-step inactivation of chromosomal genes in Escherichia coli K-12 using PCR products. Proc. Natl Acad. Sci. USA 97, 6640-6645 (2000).

24 Nishino, K. \& Yamaguchi, A. Role of histone-like protein H-NS in multidrug resistance of Escherichia coli. J. Bacteriol. 186, 1423-1429 (2004).

25 Elkins, C. A. \& Nikaido, H. Substrate specificity of the RND-type multidrug efflux pumps AcrB and AcrD of Escherichia coli is determined predominantly by two large periplasmic loops. J. Bacteriol. 184, 6490-6498 (2002).

26 Hirakawa, H. Nishino, K., Hirata, T. \& Yamaguchi, A. Comprehensive studies of drug resistance mediated by overexpression of response regulators of two-component signal transduction systems in Escherichia coli. J. Bacteriol. 185, 1851-1856 (2003).

27 Nishino, K., Nikaido, E. \& Yamaguchi, A. Regulation of multidrug efflux systems involved in multidrug and metal resistance of Salmonella enterica serovar Typhimurium. J. Bacteriol. 189, 9066-9075 (2007).

28 Nishino, K., Yamasaki, S., Hayashi-Nishino, M. \& Yamaguchi, A. Effect of NIpE overproduction on multidrug resistance in Escherichia coli. Antimicrob. Agents Chemother. 54, 2239-2243 (2010).

29 McClelland, M. et al. Complete genome sequence of Salmonella enterica serovar Typhimurium LT2. Nature 413, 852-856 (2001). 